\title{
Spatial orienting of tactile attention induced by social cues
}

\author{
SALVADOR SOTO-FARACO, SCOTT SINNETT, and AGNÈS ALSIUS \\ ICREA and Parc Científic de Barcelona, Barcelona, Spain \\ and \\ ALAN KINGSTONE \\ University of British Columbia, Vancouver, British Columbia, Canada
}

\begin{abstract}
Several studies have established that humans orient their visual attention reflexively in response to social cues such as the direction of someone else's gaze. However, the consequences of this kind of orienting have been addressed only for the visual system. We investigated whether visual social attention cues can induce shifts in tactile attention by combining a central noninformative eye-gaze cue with tactile targets presented to participants' fingertips. Data from speeded detection, speeded discrimination, and signal detection tasks converged on the same conclusion: Eye-gaze-based orienting facilitates the processing of tactile targets at the location of the gazed-at body location. In addition, we examined the effects of other directional cues, such as conventional arrows, and found that they can be equally effective. This is the first demonstration that social attention cues have consequences that reach beyond their own sensory modality.
\end{abstract}

Everyday experience reveals that humans are strongly compelled to orient their attention according to socially meaningful cues, such as the eye gaze of others. For instance, several researchers have proposed the existence of specialized brain mechanisms that are devoted to determining where other people are looking and to quickly shift attention there (Baron-Cohen, 1994; Perrett \& Emery, 1994). In line with these proposals, several findings highlight the role that spatial cues based on eye gaze play in orienting visual attention in infants (Hood, Willen, \& Driver, 1998; Maurer, 1985; Scaife \& Bruner, 1975) and adults (Driver et al., 1999; Friesen \& Kingstone, 1998; Langton \& Bruce, 1999; Ricciardelli, Baylis, \& Driver, 2000; Ricciardelli, Bricolo, Aglioti, \& Chelazzi, 2002; Ristic, Friesen, \& Kingstone, 2002).

Several recent findings have revealed that social orienting of attention extends to other types of cues, such as finger-pointing cues (see, e.g., Langton \& Bruce, 1999) and even more arbitrary (but socially meaningful) cues such as conventional arrows (Friesen, Ristic, \& Kingstone, 2004; Ristic et al., 2002; Tipples, 2002). Although there is some contention about the mechanisms that subserve orienting to biologically salient cues in comparison with more conventional cues, the attention effects triggered

This work was supported by Grants DGCYT BSO2002-01545 and TIN2004-04363-C03-02 from the Ministerio de Educación y Cultura and by Grant JSMF-20002079 from the James S. McDonnell Foundation. Correspondence concerning this article should be addressed to S. Soto-Faraco, Hospital Sant Joan de Déu (Edifici Docent), c/ Santa Rosa 39-57, Planta 4a, Esplugues de Llobregat, 08950 Barcelona, Spain (e-mail: salvador.soto@icrea.es). by these social signals have raised interesting questions regarding the classical conception of spatial attention mechanisms (see, e.g., Kingstone, Smilek, Ristic, Friesen, \& Eastwood, 2003; Langton, Watt, \& Bruce, 2000). The "standard" view, for instance, was that centrally presented cues (i.e., cues that did not bear spatial overlap with the potential target location) had to be informative as to the likely location of a target before attention would be committed to it - that is, the attentional shift to central cues was volitional rather than reflexive (Jonides, 1981; Klein, Kingstone, \& Pontefract, 1992; Posner, 1978). ${ }^{1}$

Interestingly, the consequences of orienting to socially meaningful cues do not conform strictly to either of the two classic categories of reflexive and volitional attention (see, e.g., Friesen et al., 2004; Kingstone, Friesen, \& Gazzaniga, 2000; Langton et al., 2000). Like the standard reflexive attention effect, gaze stimuli have been shown to induce rapid attentional shifts regardless of their informational value (i.e., when the cues are not predictive, and even when they are counterpredictive, of the target location; see, e.g., Driver et al., 1999; Friesen \& Kingstone, 1998; Friesen et al., 2004; Langton et al., 2000). However, as in the standard volitional effect, these cues appear at central fixation (i.e., they do not bear spatial overlap with the potential location of the target) and induce long-lasting attentional shifts. Moreover, they do not result in a period of decreased sensitivity at the attended location, as is the case for the standard investigations of the reflexive inhibition-of-return (IOR) effect (see Friesen \& Kingstone, 2003).

The goal of the present study was to address whether or not the effects of this type of attentional orienting, trig- 
gered by central nonpredictive but meaningful cues, will extend beyond the modality of vision to that of touch. There is now a wealth of evidence, derived from both the standard reflexive and the standard volitional attention paradigms, indicating that when visual attention is directed to a spatial location, the processing of visual and nonvisual stimuli alike are enhanced at that location (see Driver \& Spence, 1998, 2004, and McDonald, Driver, \& Spence, 2004, for reviews). For example, Kennett, Spence, and Driver (2002) found faster tactile judgments at the hand that had been previously cued by a nonpredictive peripheral flash than at the opposite hand (also see Gray \& Tan, 2002; Kennett, Eimer, Spence, \& Driver, 2001). Similar cross-modal effects are observed in endogenous attention, when tactile targets occurring at the location where a visual stimulus is expected are processed faster and more accurately than targets appearing at other (even more likely) locations (Spence, Pavani, \& Driver, 2000; also see Eimer \& Driver, 2000, for ERP evidence). However, the potential cross-modal effects of social cues such as eye gaze are currently unknown. Specifically, since social cues usually signal distal events, whereas touch stimuli are necessarily proximal, the extrapolation of previous visuotactile effects in spatial attention is not straightforward. However, according to current accounts, the usefulness of such gaze-induced shifts in visual attention is in allowing inferences about the object of other people's visual attention (see, e.g., Baron-Cohen, 1994; Driver et al., 1999; Langton et al., 2000). Moreover, preliminary results in our laboratory suggested that auditory spatial attention is insensitive to the direction of eye-gaze cues (Soto-Faraco, McDonald, \& Kingstone, 2002).

We used a noninformative eye-gaze cue presented at central fixation (see, e.g., Friesen \& Kingstone, 1998, 2003; Friesen et al., 2004; Kingstone et al., 2000; Kingstone, Tipper, Ristic, \& Ngan, 2004). After the cue (eyes looking laterally), a vibrotactile target was presented at varying stimulus onset asynchronies (SOAs), with equal probability at the fingertip of the cued hand and at that of the opposite (uncued) hand. We used several converging measures of target processing: a speeded simple detection task, speeded discrimination, and signal detection measures. Finally, in order to address the specificity of eye-gaze cues, we also measured the effects of arrow cues using signal detection (the strictest possible measure).

\section{EXPERIMENT 1}

\footnotetext{
Method

Participants. Seventy-five undergraduates from the University of Barcelona took part in this experiment in exchange for course credit. Each was tested in only one of the tasks (18 in the speeded detection task, 24 in the speeded discrimination task, and 33 in the signal detection task). All had normal or corrected-to-normal vision and reported normal tactile sensitivity. Data from 9 additional participants were eliminated because they erroneously responded on more than $10 \%$ of the straight-gaze trials $(n=8$; see Procedure, below), or because of a high error rate in the speeded discrimination task $(>25 \%, n=1)$.

Materials and Apparatus. We used a schematic face (see Figure 1) consisting of a black circle outline ( $96 \mathrm{~mm}$ in diameter) cen-
}

tered on the screen, containing two eyes $(12.5 \mathrm{~mm}$ to the left and right of center), a nose (3-mm dot) and a mouth (24-mm straight line). Two black circles ( $8 \mathrm{~mm}$ in diameter), representing the pupils, were drawn inside the eyes as the cue. These could be centered (straight gaze) or shifted $2.5 \mathrm{~mm}$ laterally (face looking left or right).

The tactile targets consisted of a 50-msec vibration presented at the tips of the little fingers via a bone conduction vibrator (Oticon $\mathrm{p} / \mathrm{n}$ BC461-1-100) connected to the PC soundcard. On each hand, the back of the little finger was attached to the corresponding side of the computer screen (to the left or right of the schematic face) with a Velcro strip glued to a cloth loop that, in turn, held the vibrator to the fingertip. The participants responded by using the dominant foot to depress a foot pedal placed on the floor. During the experiment, the participants wore headphones playing white noise at an intensity level that masked any noise related to the operation of the vibrators. They could use cushions under their elbows to maintain a comfortable posture. The experiment was controlled by a PC running EXPE 6 (Pallier, Dupoux, \& Jeannin, 1997).

Procedure. The participants sat in front of the computer screen (at approximately $35 \mathrm{~cm}$ ) with their little fingers attached to each side of the monitor $(16 \mathrm{~cm}$ off center, horizontally aligned with the schematic face; see Figure 1A). They were told that eye direction was uninformative as to the target location. In every trial, a face outline (without the pupils) appeared on the screen and, after $675 \mathrm{msec}$, the pupils were displayed looking right (46\%), left (46\%), or straight ahead $(8 \%)$. The participants had to refrain from responding in the latter type of trials, which were included in all tasks to ensure attention to the face. The target appeared 112,308, or 1,008 msec after the cue, and these delays constituted the SOAs. Cue direction (left, right, or straight ahead), SOA, and target location were selected randomly in each trial. Responses to the target in a straight-gaze trial resulted in an error feedback signal (three brief vibrations at both fingertips plus a text warning on the screen) to the participant. The next trial started after a randomly selected period of 2,037-2,490 msec. Each experimental task (described below) was divided into four equivalent blocks containing 78 trials each, for a total of 312 trials. To acquaint the participant with the task, a short training block (14 trials) was run prior to the test and repeated if necessary.

Speeded detection task. The target was a $50-\mathrm{msec}, 200-\mathrm{Hz}$ vibration. The participants were asked to depress the foot pedal under their toes as quickly as possible whenever the target was detected at either of the two hands. In this task only, an additional 15\% catch trials, in which no target vibration was presented, were included in order to discourage anticipatory responses.

Speeded discrimination task. The target was a 50-msec vibration at one of two possible frequencies $(340$ or $100 \mathrm{~Hz}$ ). The participants were instructed to release the foot pedal with the toe or the heel for the high- or low-frequency target, respectively. They were asked to respond as quickly as possible while maintaining accuracy.

Signal detection task. The target was a $200-\mathrm{Hz}$ vibration, the intensity of which was adjusted online (in 3-dB steps) according to a staircase designed to maintain performance between $70 \%$ and $90 \%$. On half of the trials, the target was followed by a mask (a 100-msec vibration of white noise), whereas on the other half only the mask was present. The participants were asked to respond to target-present and target-absent trials (by depressing the foot pedal with the toes and with the heel, respectively). They were instructed to respond as soon as they were sure of their decision but to prioritize accuracy over response speed. In this task, the participants received an additional training block with reduced mask intensity.

\section{Results}

Speeded detection. Responses to targets on straightgaze trials $(M=5.2 \%, S D=8)$ as well as false alarms and misses (each $<1 \%$ ) were very rare. There were no significant effects for the latter types of errors, and therefore they 
(A)

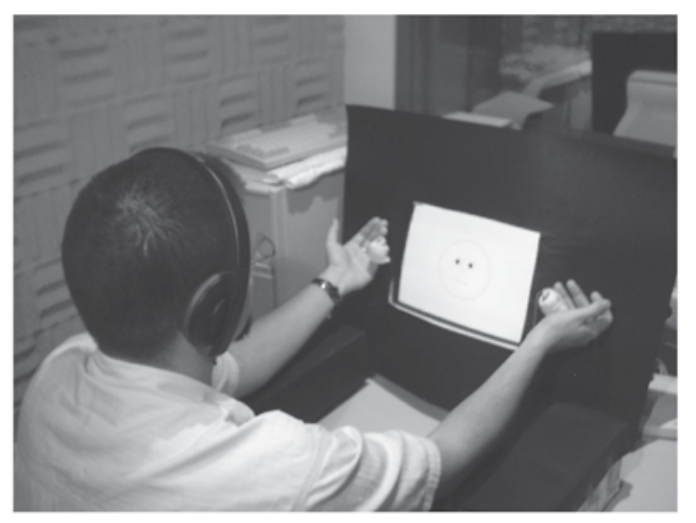

(B)



Figure 1. (A) A general view of the setup and posture adopted by the participants in all of the experiments. (B) Schematic representation of the sequence of events in Experiment 1. A schematic face (without the pupils) was presented on a fixation screen, followed by presentation of the cue (the pupils, looking left or right, are filled in). Then, after a variable amount of time (stimulus onset asynchrony) of 112, 308, or $1,008 \mathrm{msec}$, the target (represented in the figure by a vibration-like symbol) was presented to the left or right fingertip. In a proportion of the trials (no-go trials), the eves looked straight ahead. In the speeded detection task, a further type of catch trial (no target presented, not depicted in the figure) was also included in order to discourage advanced responses.

were not included in the analyses reported below. Correct response times (RTs; see Figure 2) were filtered (removing data points falling beyond two $S D$ s of the individual mean in each condition; $4.8 \%$ discarded overall, $S D=$ $1.9)$ and submitted to an ANOVA. The ANOVA included two within-participants factors: cue-target SOA (112 vs. 308 vs. $1,008 \mathrm{msec}$ ) and cuing (cued vs. uncued). The SOA effect was significant $[F(2,34)=19.7, p<.001]$, with longer RTs in the 112-msec SOA than in the 308-and 1,008 -msec SOAs (both $p \mathrm{~s}<.01$ ), reflecting a foreperiod effect (Friesen \& Kingstone, 1998; Mowrer, 1940). The RTs for the 1,008-msec SOA were longer than those for the 308-msec SOA $(p=.03)$, but the significance of this comparison is unclear when the alpha level is corrected for multiple comparisons (Bonferroni adjustment; $p<.017$ ). Moreover, this type of increase is not inconsistent with a foreperiod effect, which depends on the ability to estimate the time interval and sustain preparation for when a target is due to occur (Kingstone, 1992). Since it bears no relation to the main conclusion and did not appear in any other experiment, this difference will not be discussed further. Most importantly, however, the targets presented at the gazed-at (cued) finger were responded to faster than those presented at the nongazed-at (uncued) finger $[F(1,17)=$ $10.9, p<.005]$. Although the cuing effect reached significance only at the two shortest SOAs $(112 \mathrm{msec}, p<.05$; $308 \mathrm{msec}, p=.001 ; 1,008 \mathrm{msec}, p=.261)$, there was a numerical trend throughout all SOAs, and the interaction between cuing and SOA was not significant $(F<1)$.

Speeded discrimination (see Figure 3). The participants rarely responded on straight-gaze trials $(M=2.2 \%$, $S D=4.4)$. Target discrimination errors were relatively infrequent $(M=6.1 \%, S D=5.8)$, and their analysis revealed no main effects of SOA or cuing $(F \mathrm{~S}<1)$ and 


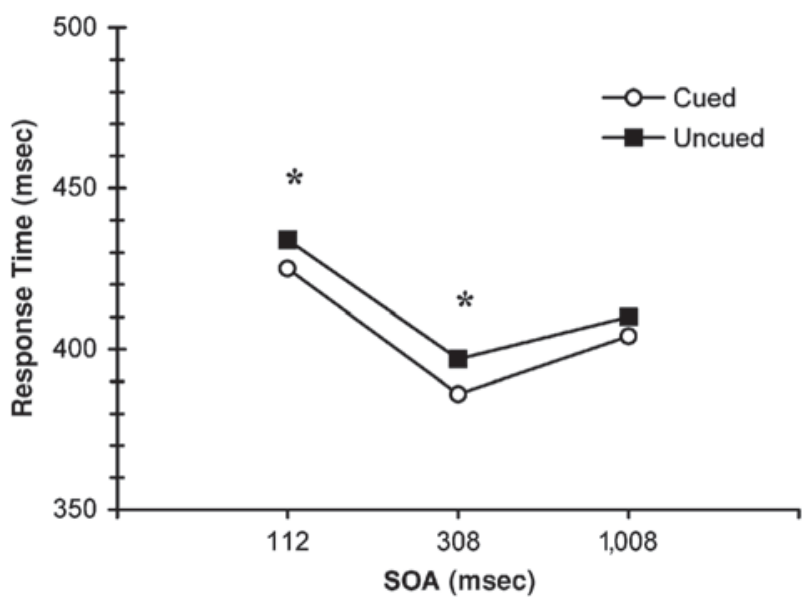

Figure 2. Results of the speeded detection task. The response latencies from target onset are presented as a function of stimulus onset asynchrony (SOA, $x$-axis) and cue (white circles, cued trials; black squares, uncued trials). The asterisks indicate significant cuing effects $(p<.05)$.

no interaction $[F(2,46)=1.4, p>.25]$. The RT analysis ( $4.4 \%$ of the data, $S D=1.8$, filtered out) revealed a main effect of SOA $[F(2,46)=85.0, p<.001]$ and, most importantly, a significant cuing effect $[F(1,23)=8.0, p=$ .009]. Since RTs to cued targets were numerically shorter than RTs to uncued targets across all SOAs, the interaction between the two factors did not approach significance $(F<1)$. However, the effect of cuing was significant only at the longest SOA (112 msec, $p=.756 ; 308 \mathrm{msec}, p=$ $.102 ; 1,008 \mathrm{msec}, p=.005)$.

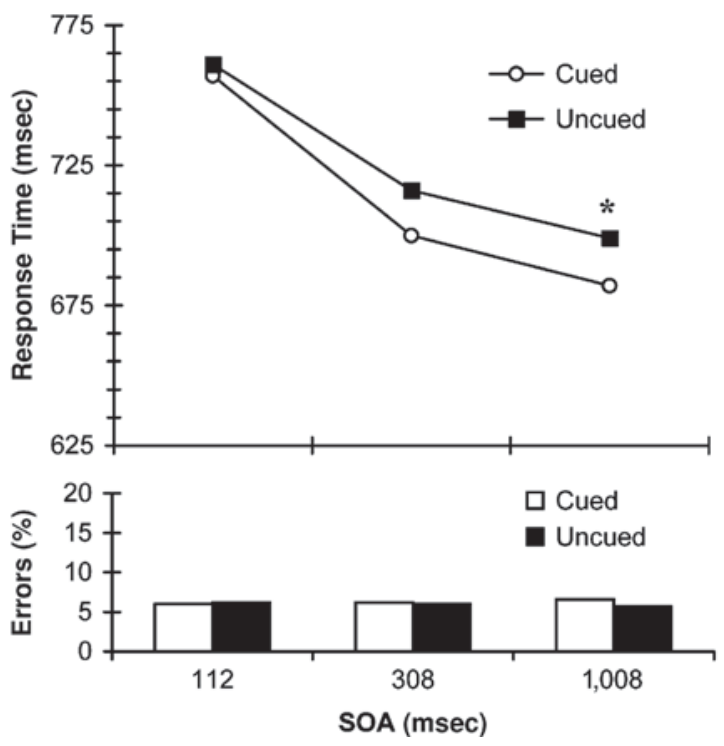

Figure 3. Results of the speeded discrimination task. The response latencies from target onset (top panel) and percentage of error (bottom panel) are presented as functions of stimulus onset asynchrony (SOA, $x$-axis) and cuing (white, cued trials; black, uncued trials). The asterisk indicates a significant cuing effect $(p<.05)$.
Signal detection (see Figure 4). The participants responded erroneously on $2.0 \%(S D=3.6)$ of the straightgaze trials. RT filtering removed $5.6 \%(S D=3.2)$ of data. The $d^{\prime}$ (sensitivity) and $c$ (criterion) parameters were calculated from the hit and false alarm rates for every participant and condition (Macmillan \& Creelman, 1991) and submitted to an ANOVA with the same factors as for the speeded detection and speeded discrimination tasks. $d^{\prime}$ did not reveal an effect of SOA $(F<1)$. Target detection was more accurate at the gazed-at location than at the opposite location, but this difference was marginal $[F(1,32)=$ $3.1, p=.085]$. There was no interaction between SOA and cuing $[F(2,64)=1.5, p>.20]$, although the cue effect approached significance only at the 1,008-msec SOA $(p=.085)$. The $c$ parameter did not reveal any significant effects (all $\left.F_{\mathrm{S}}<1\right)$. The correct RT data showed a foreperiod effect $[F(2,64)=28.6, p<.001]$ and, again, a significant cuing effect with shorter RTs at the gazed-at location than at the uncued location $[F(1,32)=24.3, p<$ $.001]$. There was no interaction $(F<1)$, and RTs on cued trials were shorter than RTs on uncued trials for each individual SOA, although the difference was significant only at the two longest SOAs (112 msec, $p=.100 ; 308 \mathrm{msec}$, $p<.05 ; 1,008 \mathrm{msec}, p<.005)$.

\section{EXPERIMENT 2}

\section{Method}

Participants. Thirty-three new participants from the same population as those in Experiment 1 were tested. Data from 6 additional participants were eliminated due to a failure to withhold responses on double-arrow (analogous to straight-gaze) trials ( $>10 \%)$.

Apparatus, Materials, and Procedure. These were exactly as in the signal detection paradigm of Experiment 1 except for the cue. The face outline was replaced with a straight line (see Figure 5A), and the pupils were replaced with two arrowheads that appeared $12.5 \mathrm{~mm}$ to the left and right of center, both pointing either to the left or to the right. As a replacement of the straight-gaze trials, some trials contained the left and right arrowheads superimposed, forming a cross at each of the two points on the straight line.

\section{Results}

The data (see Figures 5B and 5C) were filtered as in Experiment $1(3.2 \%$ of data removed, $S D=1.8)$. Erroneous responses on the straight-gaze trials amounted to $2.3 \%(S D=2.7)$. An ANOVA on the $d^{\prime}$ scores revealed no effect of SOA $(F<1)$ or cuing $[F(1,32)=1.0, p=$ $.320]$ and no significant interaction $(F<1)$. The ANOVA on the criterion parameter did not reveal any significant effects either (all $F \mathrm{~s}<1$ ). The ANOVA on correct RTs revealed an effect of SOA $[F(2,64)=7.9, p=.001]$ and a significant cuing effect $[F(1,32)=13.5, p=.001]$, with shorter RTs at the cued location. Although there was no interaction between SOA and cuing $(F<1)$, the numerical advantage observed in the cued trials was significant at the two longest SOAs (112 msec, $p=.256 ; 308 \mathrm{msec}$, $p<.05 ; 1,008 \mathrm{msec}, p<.005)$.

We pooled the data from the signal detection paradigm of Experiment 1 and Experiment 2 and submitted them to new analyses (on RTs and $d^{\prime}$ ), including experiment as 


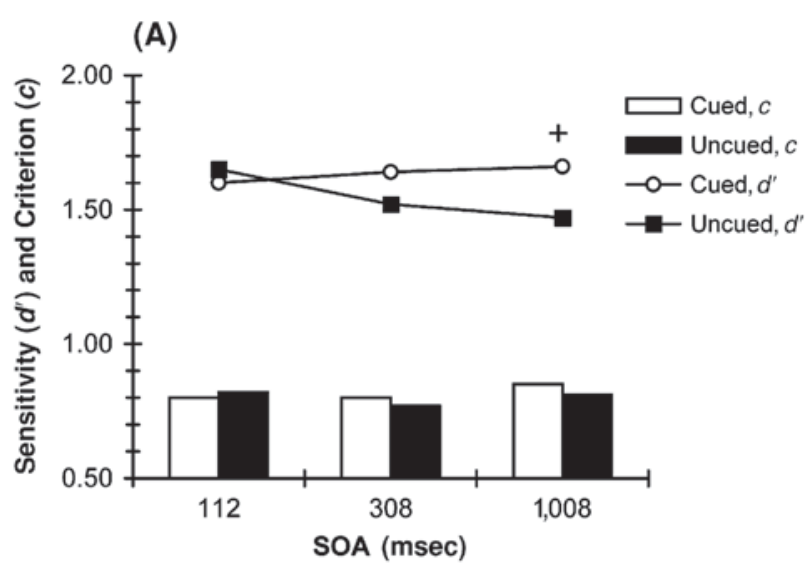

(B)

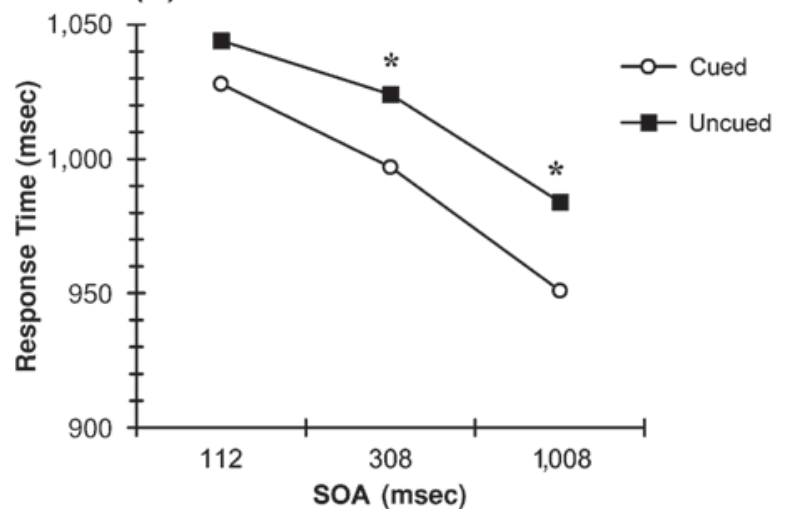

Figure 4. Results of the signal detection task in Experiment 1. Sensitivity $\left(d^{\prime}\right)$ and criterion $(c)$ parameters $(\mathrm{A})$ and the response latencies from cue onset $(B)$ are presented as functions of stimulus onset asynchrony (SOA, $x$-axis) and cue (white, cued trials; black, uncued trials). The asterisks indicate significant cuing effects $(p<.05)$, and the plus sign indicates a marginally significant difference $(.05<p<.1)$.

a between-participants factor. In terms of RTs, the main effects of experiment [faster responses in Experiment 1 than in Experiment 2; $F(1,64)=29.7, p<.001]$, SOA $[F(2,128)=32.9, p<.001]$, and cuing $[F(1,64)=37.0$, $p<.001]$ were significant. The only interaction to reach significance was experiment $\times \operatorname{SOA}[F(2,128)=6.6$, $p<.005$ ], due to a greater decrease in RTs with increasing SOA in Experiment 2. In terms of $d^{\prime}$, the ANOVA revealed a significant main effect of cuing $[F(1,64)=$ $4.0, p<.05]$, whereas all other effects and interactions remained far from significant (all $F_{\mathrm{s}}<1$ ).

\section{GENERAL DISCUSSION}

The main conclusion to emerge from this study is that the effect of visual social cues to spatial location transcends the visual domain, enhancing the processing of tactile stimuli at the cued body location. We have shown, across three different tasks, that one's tactile attention shifts to the location toward which the eyes of a schematic face are looking. Moreover, the results of Experiment 2 indicate that, in principle, there seems to be little difference between the behavioral effects of eye-gaze cues and those of other (perhaps less biologically relevant) directional cues, such as arrows. Since the cues used in this study were central and noninformative, the resulting tactile attention effect appears to be both fundamental and reflexive in nature. Indeed, this effect follows a time course similar to that observed with visual targets used in similar paradigms - that is, the gaze effect appears rapidly and does not lead to IOR.

Despite the consistent lack of interaction between SOA and cuing, which suggests a rapid and persistent shift of attention, individual tests at each SOA as well as visual inspection of the data demand some caution in drawing this conclusion. For example, in the speeded discrimination and signal detection tasks it would appear that tactile processing benefited from the attention shift only after the 112-msec SOA. It could be that, because of the small size of the effects at the longer SOAs, there was simply not enough statistical power in each individual experiment to detect the interaction. However, further data analyses counter this possible interpretation; even when the RT data of all 108 participants are pooled, the SOA $\times$ cuing interaction remained far from significant $[F(2,208)=1.66$, $p=.193]$. Moreover, the effect of cuing at the 112-msec SOA in this pooled analysis was significant $[F(1,104)=$ $5.2, p<.025]$, without a hint of interaction between task and SOA $(F<1)$. We argue, therefore, that there was a consistent albeit occasionally small cuing effect throughout, a result that is consistent with the reflexive nature of the attention shift as reported for visual targets (Kingstone et al., 2003).

The use of several converging measures helps us to determine that these effects are attentional in nature. On the basis of speeded simple detection data alone, one could reasonably argue that detection RTs to a cued location are shorter merely because of a response criterion shift. However, speeded discrimination and signal detection data clearly show that our participants were not trading faster response speed for poorer response accuracy and that there was no shift in response criterion. Consequently, the present results demonstrate for the first time that central noninformative cues can be effective at triggering shifts in tactile attention.

Despite the claims of some researchers that eye-gaze cues are special in terms of triggering attention shifts, the pattern of effects obtained here with arrow cues was fairly comparable to that obtained using eye-gaze cues. The only discrepancy was the existence of a nearly significant effect of gaze cues on sensitivity, which was not seen when arrow cues were used. However, this difference can hardly be taken as a basis for dissociation, given the similar profile for RT and the fact that the interaction between experiment and cuing was far from significant (as was the interaction between experiment and cuing at every SOA level; all $p \mathrm{~s}>.25$ ). Therefore, arrows seem to be just as effective as eye gaze in orienting tactile attention. This is 
(A)

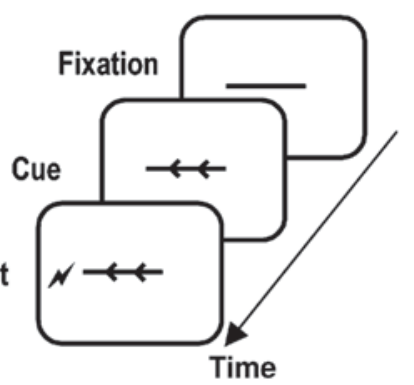

(B)

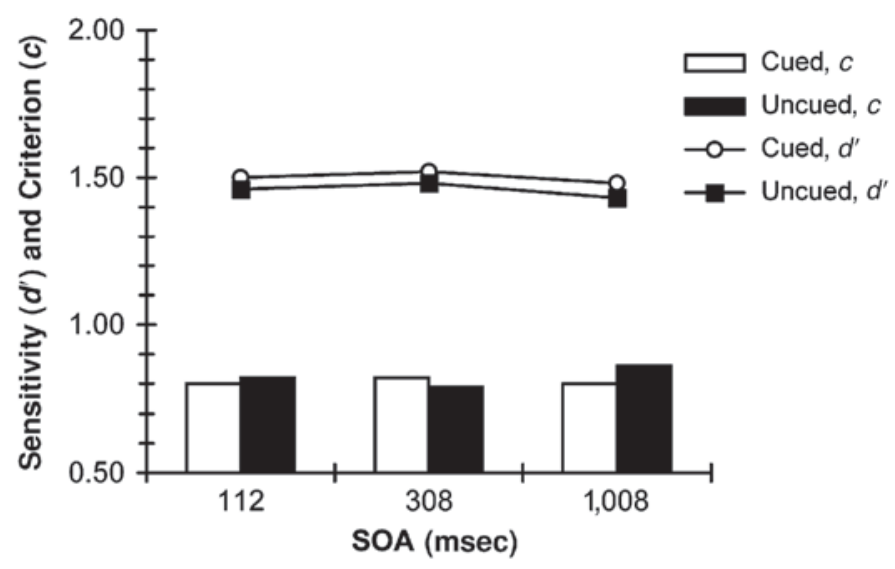

(C)

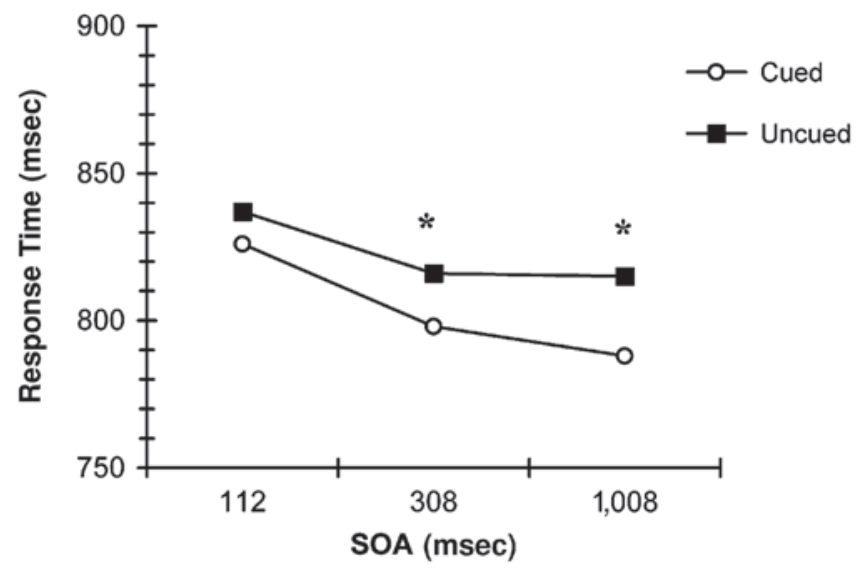

Figure 5. (A) A schematic representation of the sequence of events in a cued trial in the signal detection task used in Experiment 2. The sensitivity $\left(d^{\prime}\right)$ and criterion $(c)$ averages (B) and the response latencies from cue onset $(C)$ are presented as functions of stimulus onset asynchrony (SOA, $x$-axis) and cuing (white, cued trials; black, uncued trials). The asterisks indicate significant cuing effects $(p<.05)$.

consistent with other recent findings, in which no such dissociation has been observed in terms of behavioral results (see, e.g., Ristic et al., 2002).

However, this lack of behavioral dissociation does not necessarily imply that eye gaze and other types of cues are processed alike (Kingstone et al., 2000; Kingstone et al., 2004) or even that they always have an equivalent behav- ioral effect. For instance, Friesen et al. (2004) have found that it is harder to attend to the opposite direction of a gaze cue than to that of an arrow cue (also see Kingstone et al., 2000, and Ricciardelli, Ro, \& Driver, 2002, for laterality-based dissociations). Moreover, evidence from neuroimaging studies points to certain brain areas (i.e., the superior temporal sulcus) as being specialized in de- 
coding dynamic aspects of faces, such as eye-gaze direction (Hoffman \& Haxby, 2000; Kingstone et al., 2004; see Perrett \& Emery, 1994, for evidence from neurophysiology studies). We argue that despite the potentially special processing that eye-gaze cues may undergo, their effects on spatial attention may often be equivalent to those of other types of directional cues. This is consistent with a proposed circuit in which first the cue would be decoded by a specialized mechanism and then the output of this processing would be relayed to a generic attention mechanism, which would reorient the focus of attention (see Corbetta \& Shulman, 2002, for a recent review of the possible structural and functional components of attentional orienting). In line with this idea, extant neuroimaging data has revealed links between the superior temporal sulcus (putatively the eye-gaze interpreter) and the posterior parietal cortex, an area critical for orienting attention in space (George, Driver, \& Dolan, 2001; also see Kingstone et al., 2004). Interestingly for the interpretation of our results, several neuropsychological (e.g., Làdavas \& Farnè, 2004), human electrophysiological (e.g., Kingstone et al., 2004; McDonald, Teder-Sälejärvi, Di Russo, \& Hillyard, 2003), and neuroimaging (e.g., Macaluso \& Driver, 2004) studies show that the cited attention mechanisms in the parietal cortex are multimodal.

In conclusion, we have shown that the consequences of uninformative central, social cues on the spatial allocation of attention can span to other sensory modalities. This extends previous cross-modal attention findings using standard (exogenous or endogenous) attention paradigms. The pattern of results suggests a rapid and sustained attention shift that is not followed by IOR. Finally, this extension to other modality combinations appears to be equivalent across eye-gaze and arrow cues, a finding that supports the notion that the attention shift, but not necessarily the interpretation of the cue, may be subserved by a common multimodal mechanism.

\section{REFERENCES}

Baron-Cohen, S. (1994). How to build a baby that can read minds: Cognitive mechanisms in mindreading. Cahiers de Psychologie Cognitive, 13, 513-552.

Corbetta, M., \& Shulman, G. L. (2002). Control of goal-directed and stimulus-driven attention in the brain. Nature Reviews Neuroscience, 3, 201-215.

Driver, J., Davis, G., Ricciardelli, P., Kidd, P., Maxwell, E., \& BARON-CoHEN, S. (1999). Gaze perception triggers visuospatial orienting by adults in a reflexive manner. Visual Cognition, 6, 509-540.

Driver, J., \& Spence, C. (1998). Attention and the crossmodal construction of space. Trends in Cognitive Sciences, 2, 254-262.

Driver, J., \& Spence, C. (2004). Crossmodal spatial attention: Evidence from human performance. In C. Spence \& J. Driver (Eds.), Crossmodal space and crossmodal attention (pp. 179-220). Oxford: Oxford University Press.

EIMER, M., \& Driver, J. (2000). An event-related brain potential study of cross-modal links in spatial attention between vision and touch. Psychophysiology, 37, 697-705.

Friesen, C. K., \& Kingstone, A. (1998). The eyes have it! Reflexive orienting is triggered by nonpredictive gaze. Psychonomic Bulletin \& Review, 5, 490-495.

Friesen, C. K., \& Kingstone, A. (2003). Abrupt onsets and gaze direc- tion cues trigger independent reflexive attentional effects. Cognition, 87, B1-B10.

Friesen, C. K., Ristic, J., \& Kingstone, A. (2004). Attentional effects of counterpredictive gaze and arrow cues. Journal of Experimental Psychology: Human Perception \& Performance, 30, 319-329.

George, N., Driver, J., \& Dolan, R. J. (2001). Seen gaze-direction modulates fusiform activity and its coupling with other brain areas during face processing. NeuroImage, 13, 1102-1112.

Gray, R., \& TAN, H. Z. (2002). Dynamic and predictive links between touch and vision. Experimental Brain Research, 145, 50-55.

Hoffman, E. A., \& HaXby, J. V. (2000). Distinct representations of eye gaze and identity in the distributed human neural system for face perception. Nature Neuroscience, 3, 80-84.

Hood, B. M., Willen, J. D., \& Driver, J. (1998). Adult's eyes trigger shifts of visual attention in human infants. Psychological Science, $\mathbf{9}$, 131-134.

JONIDES, J. (1981). Voluntary versus automatic control over the mind's eye's movement. In J. Long \& A. Baddeley (Eds.), Attention and performance IX (pp. 187-203). Hillsdale, NJ: Erlbaum.

Kennett, S., Eimer, M., Spence, C., \& Driver, J. (2001). Tactile-visual links in exogenous spatial attention under different postures: Convergent evidence from psychophysics and ERPs. Journal of Cognitive Neuroscience, 13, 462-478.

Kennett, S., Spence, C., \& Driver, J. (2002). Visuo-tactile links in covert exogenous spatial attention remap across changes in unseen hand posture. Perception \& Psychophysics, 64, 1083-1094.

Kingstone, A. (1992). Combining expectancies. Quarterly Journal of Experimental Psychology, 44A, 69-104.

Kingstone, A., Friesen, C. K., \& Gazzaniga, M. S. (2000). Reflexive joint attention depends on lateralized cortical connections. Psychological Science, 11, 159-166.

Kingstone, A., Smilek, D., Ristic, J., Friesen, C. K., \& Eastwood, J. D. (2003). Attention, researchers! It is time to take a look at the real world. Current Directions in Psychological Science, 12, 176-180.

Kingstone, A., Tipper, C., Ristic, J., \& NGan, E. (2004). The eyes have it! An fMRI investigation. Brain \& Cognition, 55, 269-271.

Klein, R. M., Kingstone, A., \& Pontefract, A. (1992). Orienting of visual attention. In K. Rayner (Ed.), Eye movements and visual cognition: Scene perception and reading (pp. 46-67). New York: Springer.

LÀDAVAS, E., \& FARNÈ, A. (2004). Neuropsychological evidence of integrated multisensory representation of space in humans. In G. Calvert, C. Spence, \& B. Stein (Eds.), The handbook of multisensory processes (pp. 799-818). Cambridge, MA: MIT Press.

Langton, S. R. H., \& BRUCE, V. (1999). Reflexive visual orienting in response to the social attention of others. Visual Cognition, 6, 541-567.

Langton, S. R. H., Watt, R. J., \& Bruce, V. (2000). Do the eyes have it? Cues to the direction of social attention. Trends in Cognitive Sciences, 4, 50-59.

Macaluso, E., \& Driver, J. (2004). Functional imaging evidence for multisensory spatial representations and cross-modal attentional interactions in the human brain. In G. A. Calvert, C. Spence, \& B. E. Stein (Eds.), The handbook of multisensory processes (pp. 529-548). Cambridge, MA: MIT Press.

Macmillan, N., \& Creelman, C. (1991). Detection theory: A user's guide. Cambridge: Cambridge University Press.

MaURER, D. (1985). Infant's perception of facedness. In T. Field \& N. Fox (Eds.), Social perception in infants (pp. 73-100). Norwood, NJ: Ablex.

McDonald, J. J., Driver, J., \& SPence, C. (2004). Exogenous spatialcuing studies of human crossmodal attention and multisensory integration. In C. Spence \& J. Driver (Eds.), Crossmodal space and crossmodal attention (pp. 277-320). Oxford: Oxford University Press.

McDonald, J. J., Teder-Sälejärvi, W. A., Di Russo, F., \& Hillyard, S. A. (2003). Neural substrates of perceptual enhancement by crossmodal spatial attention. Journal of Cognitive Neuroscience, 15, 10-19.

Mowrer, O. H. (1940). Preparatory set (expectancy): Some methods of measurement. Psychological Monographs, 52(2, Whole No. 233).

Pallier, C., Dupoux, E., \& Jeannin, X. (1997). EXPE: An expandable programming language for on-line psychological experiments. Behavior Research Methods, Instruments, \& Computers, 29, 322-327. 
Perrett, D. I., \& Emery, N. J. (1994). Understanding the intentions of others from visual signals: Neuropsychological evidence. Cahiers de Psychologie Cognitive, 13, 683-694.

Posner, M. I. (1978). Chronometric explorations of mind. Hillsdale, NJ: Erlbaum.

Ricciardelli, P., BAylis, G., \& Driver, J. (2000). The positive and negative of human expertise in gaze perception. Cognition, 77, B1-B14

Ricciardelli, P., Bricolo, E., Aglioti, S. M., \& Chelazzi, L. (2002). My eyes want to look where your eyes are looking: Exploring the tendency to imitate another individual's gaze. NeuroReport, 13, 22592264.

Ricciardelli, P., Ro, T., \& Driver, J. (2002). A left visual field advantage in perception of gaze direction. Neuropsychologia, 40, 769-777.

Ristic, J., Friesen, C. K., \& Kingstone, A. (2002). Are eyes special? It depends on how you look at it. Psychonomic Bulletin \& Review, 9, 507-513.

Scaife, M., \& Bruner, J. S. (1975). The capacity of joint visual attention in the infant. Nature, 253, 265-266.

Soto-Faraco, S., McDonald, J., \& Kingstone, A. (2002, April). Gaze direction: Effects on attentional orienting and crossmodal target re- sponses. Paper presented at the Ninth Annual Meeting of the Cognitive Neuroscience Society, San Francisco.

Spence, C., Pavani, F., \& Driver, J. (2000). Crossmodal links between vision and touch in covert endogenous spatial attention. Journal of Experimental Psychology: Human Perception \& Performance, 26, 1298-1319.

Tipples, J. (2002). Eye gaze is not unique: Automatic orienting in response to uninformative arrows. Psychonomic Bulletin \& Review, 9, 314-318.

\section{NOTE}

1. Reflexive orienting is the result of a stimulus-driven process whereby the focus of attention is automatically drawn toward a salient event (such as a sudden onset in the periphery), regardless of the informative nature of the cue and without voluntary control.

(Manuscript received September 17, 2004; revision accepted for publication March 13, 2005.) 